\title{
GPS Signal Detection under Multiplicative and Additive Noise
}

\author{
P. Huang ${ }^{1}, \mathrm{Y} . \mathrm{Pi}^{1}$ and I. Progri ${ }^{2}$ \\ ${ }^{1}$ (University of Electronic Science and Technology of China University, \\ Chengdu, China) \\ ${ }^{2}$ (Giftet Inc., Worcester, MA USA) \\ (E-mail: pdhuang2007@gmail.com)
}

\begin{abstract}
In some Global Positioning System (GPS) signal propagation environments, especially in the ionosphere and urban areas with heavy multipath, GPS signal encounters not only additive noise but also multiplicative noise. In this paper we compare and contrast the conventional GPS signal acquisition method which focuses on handling GPS signal acquisition with additive noise, with the enhanced GPS signal processing under multiplicative noise by proposing an extension of the GPS detection mechanism, to include the GPS detection model that explains detection of the GPS signal under additive and multiplicative noise. For this purpose, a novel GPS signal detection scheme based on high order cyclostationarity is proposed. The principle is introduced, the GPS signal detection structure is described, the ambiguity of initial PseudoRandom Noise (PRN) code phase and Doppler shift of GPS signal is analysed. From the simulation results, the received GPS signal at low power level, which is degraded by additive and multiplicative noise, can be detected under the condition that the received block of GPS data length is at least $1.6 \mathrm{~ms}$ and sampling frequency is at least $5 \mathrm{MHz}$.
\end{abstract}

\section{KEYWORDS}

1. Weak GPS Signal Acquisition. 2. Multiplicative Noise. $\quad 3$. Doppler shift. 4. Cyclostationarity.

Submitted: 5 August 2012. Accepted: 5 October 2012. First published online: 20 November 2012.

1. INTRODUCTION. Weak Global Positioning System (GPS) signal acquisition is a widely studied issue in satellite navigation signal processing where the obstructed environments is illustrated in Figure 1. Psiaki (2011) has proposed improving weak GPS signal acquisition performance by accumulating received data to increase signal to noise ratio. The algorithm of piling up GPS signals is in principle, direct and effective, and is tested in the simulation. Huang et al., (2009) has proposed a GPS signal detection algorithm employing Duffing chaotic oscillator to detect weak GPS signals, which utilizes the immunity to noise and sensitivity to periodical signals. Madhani et al., (2003) exploits the inter-symbol-interference between different GPS PseudoRandom Noise (PRN) codes to improve GPS signal acquisition performance. 


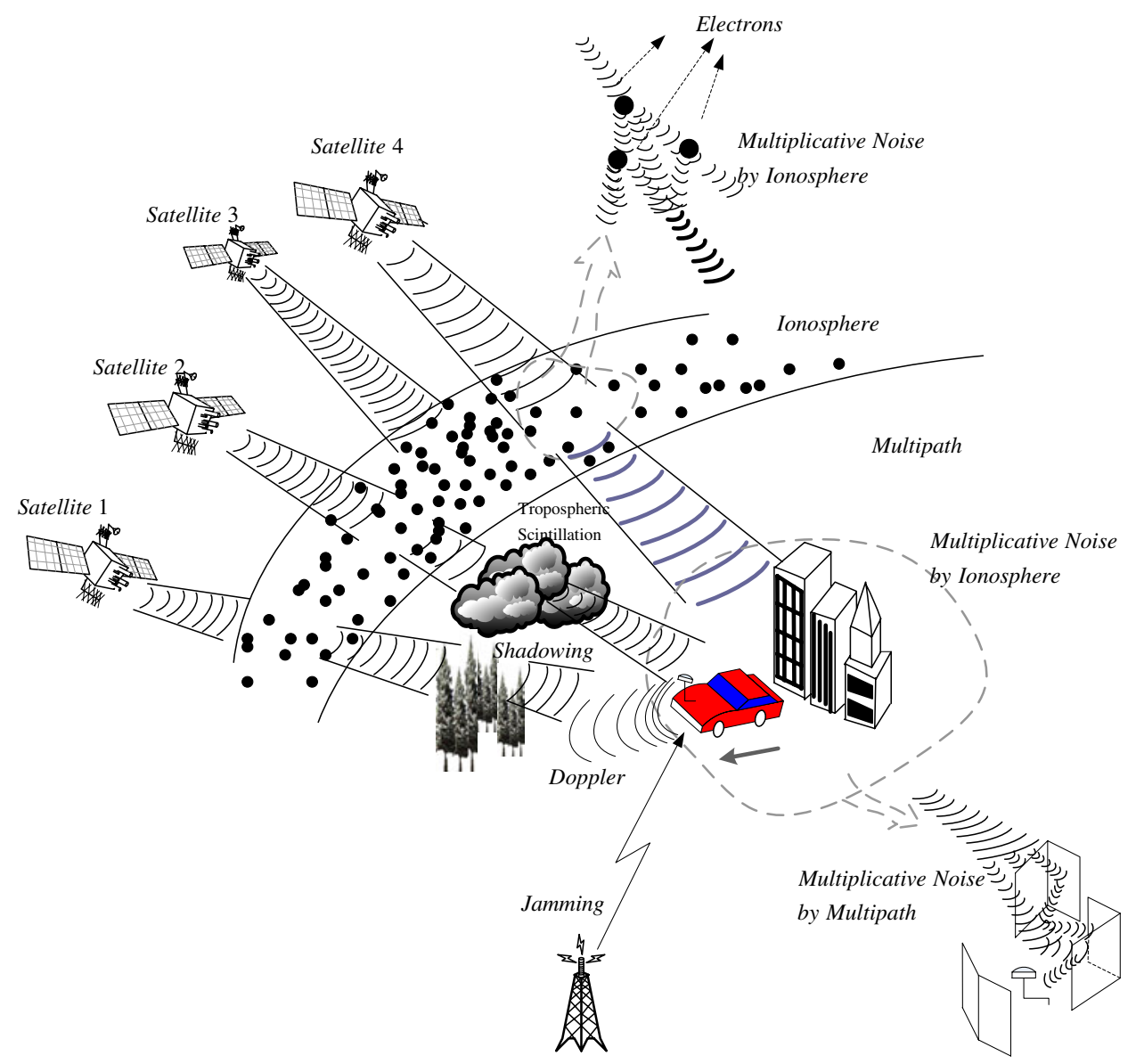

Figure 1. An illustration of the multiplicative noise environment/received GPS signal.

Progri et al., (2006) proposed to enhance the acquisition process by including the estimation of Doppler along with the estimation of the Time-Of-Arrival (TOA).

The existing methods of the weak GPS signal acquisition are effective in both simulations and experiments. However, the noise models in the received GPS signal are almost assumed to be additive almost with no exception.

However, closer observations and investigations from Zaikin et al., (2002), Middleton (1999) and Lee et al., (2008) have revealed that the received GPS signal contains multiplicative noise in addition to additive noise. Song and Uhm (1991) studied the method of detecting signal under multiplicative noise and additive noise; Song and Kassam (1990) presented the diagram block describing the generalized noise model. In the study of Zaikin et al., (2002), a generalized signal form in communications is used, which is called the bistable system. According to Zaikin et al., (2002), the phase transition at extremely high frequency causes the noise which can be modelled as multiplicative noise. Meanwhile, the additive noise is the amplitude disturbance caused by any kind of outside effects.

Figure 1 illustrates the degraded GPS signal model under multiplicative noise and additive noise. In Figure 1, there are quite a lot of factors which induce GPS signal 
fading, Doppler, evil waveform spurious transmissions, shadowing (foliage etc.,), signal jamming, antenna and receiver effects, ionospheric scintillations, tropospheric scintillation, high dense multipath, etc., (Progri 2003, 2011, 2013; Progri et al., 2006; Ma et al., 2001). Doppler shift degrades the GPS signal continuously due to the GPS satellite motion. The Doppler shift will induce a huge correlation loss when acquiring the GPS signal. 'Evil' waveforms are GPS signals that have a distorted PRN code modulation waveform (MacGougan et al., 2001). Such a deformation can be modelled by a lead or a lag of the rising or falling edges of the modulation code, and/or by a second-order filtering of this waveform (Macabiau, 2000). Huge objects, like walls of buildings, roofs of the wooden houses and the foliage etc., will block the transmission of GPS signals from satellites to receivers. Many other electromagnetic waves exist, and the GPS signals will inevitably be jammed by signals of different modulation types, carrier frequencies and transmission powers. The troposphere also degrades the GPS signal; this effect is caused by tropospheric water particles. Such effects caused by tropospheric scintillation can be modelled as the decrease in the GPS signal amplitude and time delay (Progri, 2003; Progri et al., 2006).

Degradation effects have been widely studied and so have good and successful approximation models. Indeed, the electrons in the ionosphere are of high density. When GPS signal passes through the ionosphere, each electron will cause a phase change in the carrier. The carrier phase fluctuation caused by the abundant electrons can be modelled as a multiplicative noise process. However, in normal weather conditions (e.g., sunny days) the degradation caused by multiplicative noise is not severe. The experimental results are illustrated in Figure 3(b), in which GPS data were gathered on sunny days.

In Figure 1, high dense multipath-inducing multiplicative noise is also illustrated. As we know, each path induces a different change effect on the GPS carrier phase. The comprehensive carrier phase changing effect in a huge number of paths can also be modelled as the multiplicative noise. Moreover, the additive and multiplicative noise in the GPS signals has been modelled (Progri et al., 2006).

However, there is little discussion on how to acquire GPS signals degraded by both multiplicative and additive noises. In this article, GPS signal acquisition in the environments with multiplicative and additive noise is analysed, discussed, modelled, and simulated.

2. NECESSITY OF STUDYING EFFECTS FROM MULTIPLICATIVE AND ADDITTIVE NOISE ON GPS SIGNAL. If the GPS signal can reach the GPS receiver directly, the received GPS signal power level is about $-130 \mathrm{dBm}$ (Tsui, 2004) and the receiver's background noise power is $-174 \mathrm{dBm} / \mathrm{Hz}$. In high Signal to Noise Ratio (SNR) conditions the GPS signal can be successfully acquired using the traditional receiver structure described. Such a statement can be verified by experimental results in (Psiaki, 2001; Tsui, 2004).

Figure 2 illustrates the GPS signal channel model with multiplicative and additive noise. In Figure 2 the left part illustrates the GPS signal model which considers additive and multiplicative noise. The different blocks with dash lines represent different GPS satellites in view. As described in Figure 2, the signal from each satellite encounters multiplicative noise $\varepsilon *(t)$ inevitably induced by electrons in the ionosphere. The strength of the multiplicative noise in the model depends on whether the signal 


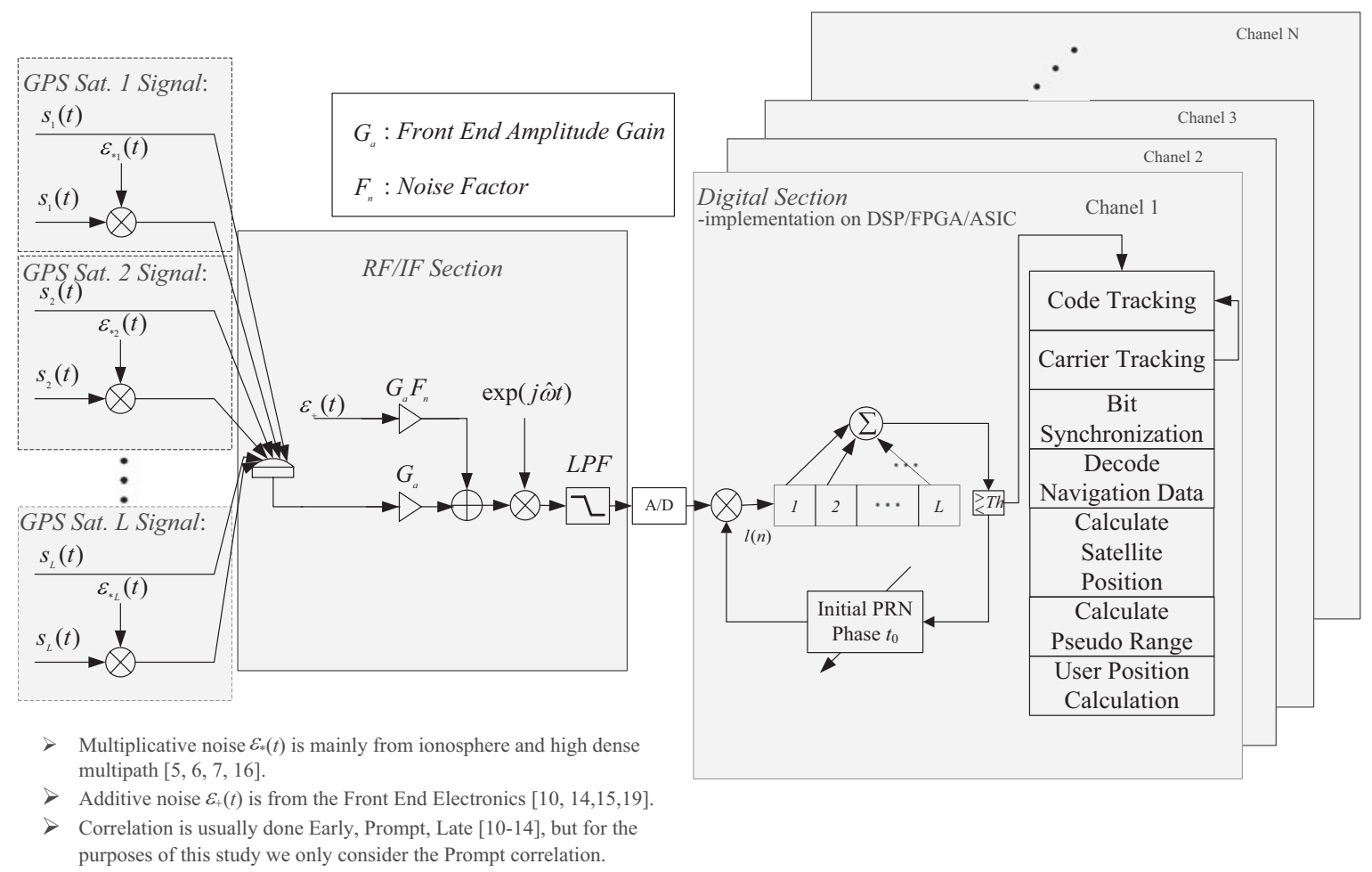

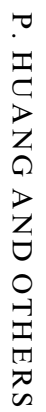

Figure 2. Block diagram of GPS signal acquisition based on correlation. 

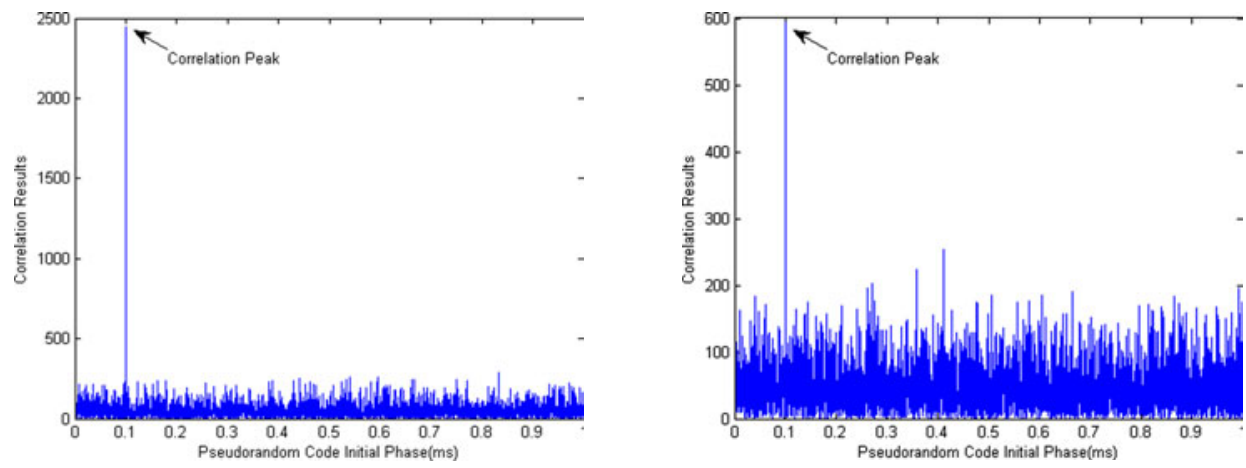

Correlation result under multiplicative noise at (a) $-184 \mathrm{dBm} / \mathrm{Hz}$ (b) $-169.2 \mathrm{dBm} / \mathrm{Hz}$
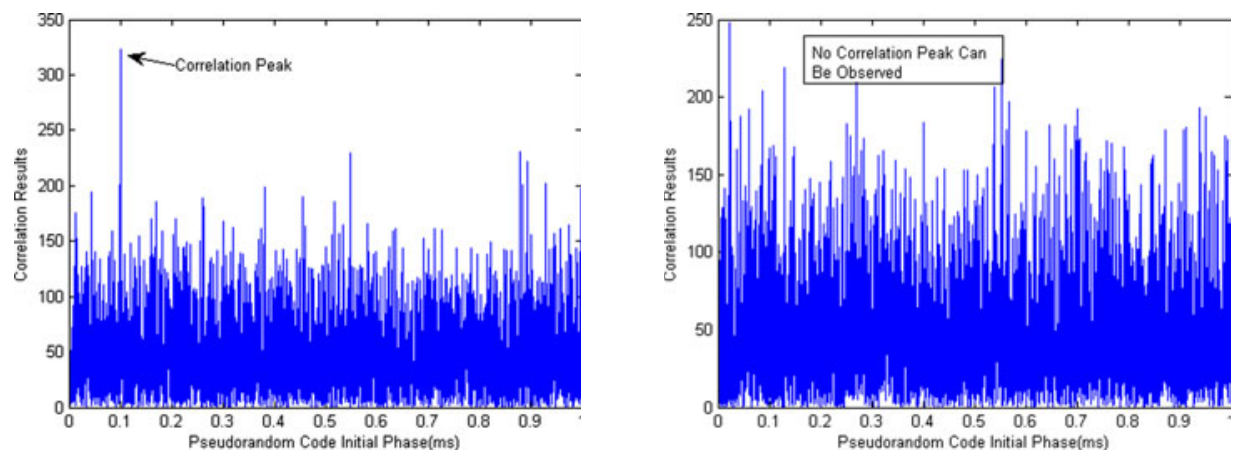

Correlation result under multiplicative noise at (c) $-167.9 \mathrm{dBm} / \mathrm{Hz}(\mathrm{d})-167 \mathrm{dBm} / \mathrm{Hz}$

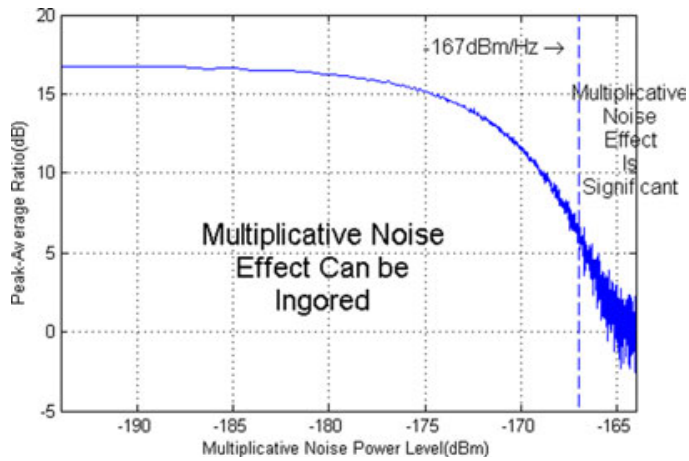

(e) Peak-to-average ratio versus multiplicative noise

Figure 3. Degradation effects from multiplicative noise on GPS signal correlation peak-average ratio.

encounters high density multipath effects. The additive noise $\varepsilon_{+}(t)$ is mainly caused by the receiver electronics in the front-end, which is thus model in the Radio Frequency/ Intermediate Frequency (RF/IF) section.

As the explanation for Figure 1 stated in Section 1, the factors which induce multiplicative noise in the received GPS signal are the severe carrier phase distortions caused by the abundant electrons in the ionosphere and high dense multipath in challenging environments, such as urban streets. 
Indeed, the multiplicative noise is usually ignored, which is reasonable. The preliminary simulation results is provided in Figure 3, which aims at demonstrating the reasonability for ignoring multiplicative noise in the condition of strong signal strength.

Figure 3 illustrates correlation results under different multiplicative noise at different multiplicative noise levels. As Psiaki (2001) and Tsui (2004) suggest, the background noise level is $-174 \mathrm{dBm} / \mathrm{Hz}$ in normal conditions; hence, in the simulation results in Figure 3, the additive noise takes $-174 \mathrm{dBm} / \mathrm{Hz}$ as the fixed value. After that, we modify the variance of multiplicative noise on the carrier phase of the GPS signal. The readers are reminded that the referable number of noise residing on GPS signal carrier phase has not been found. Therefore, we have assumed the multiplicative noise varies in the range from $-195 \mathrm{dBm} / \mathrm{Hz}$ to $-165 \mathrm{dBm} / \mathrm{Hz}$ as the indicated by the $x$-axis in Figure $3 \mathrm{e}$. In Figures $3 \mathrm{a}$ to $3 \mathrm{~d}$, the correlation results under multiplicative noise at different multiplicative noise power levels are presented. In Figure 3e, when multiplicative noise is larger than $-162 \mathrm{dBm} / \mathrm{Hz}$, the correlation results between the received GPS signal and the local replica decrease more than $13 \mathrm{~dB}$. In Figure 3d, when encountering multiplicative noise at about $-167 \mathrm{dBm} / \mathrm{Hz}$, the traditional GPS signal acquisition method based on correlation is not able to acquire the GPS signal. Therefore, the partial conclusion can be drawn that if the multiplicative noise residing on the carrier phase is smaller than $-167 \mathrm{dBm} / \mathrm{Hz}$, the GPS signal can be detected from the background noise.

Thus, we can see the GPS receiver can detect GPS signals arriving at the receiver without obstruction. In those conditions the noise caused by the ionosphere and troposphere will not degrade the GPS signal acquisition performance severely.

However, in challenging environments such as 'downtown' areas, GPS signal degrades extremely severely and the signal envelope varies very fast. As mentioned in Section 1, the multiplicative noise becomes very strong in urban environments due to high density multipaths which corresponds to the right part from the dashed line in Figure 3e. Therefore, it is necessary to study the effect of multiplicative noise on the received GPS signal in environments with high density multipaths.

\section{CYCLOSTATIONARITY CHARACTERISTICS OF THE GPS} SIGNAL UNDER MULTIPLICATIVE AND ADDITIVE NOISE.

3.1. Cyclostationary Feature of Ideal GPS Signal. In this subsection, the cyclostatistics will be introduced, taking into consideration specific properties of the GPS signal. Suppose $c(k)$ denotes the Coarse Acquisition (C/A) code. The mean value of C/A code is defined below:

$$
M_{C A}=\frac{1}{1023} \sum_{k=1}^{1023} c(k)= \pm 1 / 1023
$$

Since $1 / 1023 \ll 1$, the expected mean value of C/A code approximates to 0 .

The autocorrelation function of the GPS signal is presented below:

$$
R_{G P S}(t, \tau)=\int_{t}^{t+\Delta t} x(t) x(t+\tau) d t
$$

The GPS signal $x(t)$ is periodic with period $T_{0}\left(T_{0}=1 \mathrm{~ms}\right)$ as:

$$
x(t)=x\left(t+T_{0}\right)
$$


Therefore, from Equation (3) we have:

$$
R_{G P S}\left(t+T_{0}, \tau\right)=\left\{\begin{array}{c}
\int_{t}^{t+\Delta t} x\left(t+T_{0}\right) x\left(t+T_{0}+\tau\right) d t \\
=\int_{t}^{t+\Delta t} x(t) x(t+\tau) d t
\end{array}=R_{G P S}(t, \tau)\right.
$$

As the mean value of the GPS signal is constant and the autocorrelation function is periodic versus time, the GPS signal is cyclostationary according to the definition by (Gardner, 1994; 2006).

Because of the periodicity in the GPS signal autocorrelation function, the Fourier series of the periodic function can be calculated as:

$$
R_{G P S}(t, \tau)=\sum_{n=-\infty}^{+\infty} R_{G P S}^{n / T_{0}}(\tau) e^{j 2 \pi^{n} / T_{0}^{t}}
$$

where:

$$
R_{G P S}^{n / T_{0}}(\tau)=\int_{0}^{\Delta t} R_{G P S}(t, \tau) e^{-j 2 \pi^{n} / T_{0}^{t}} .
$$

$n$ is positive integer.

Defining $\alpha$ as the cyclic frequency by $\alpha=n / T_{0}$; then, $R_{G P S}^{\alpha}(\tau)$ is the cyclicautocorrelation function of cyclic frequency $\alpha$.

$S_{G P S}^{\alpha}(f)$ is the cyclic spectrum which is the Fourier transformation of $R_{G P S}^{\alpha}(\tau)$.

$$
S_{G P S}^{\alpha}(f)=\int R_{G P S}^{\alpha}(\tau) e^{-j 2 \pi f t} d \tau
$$

After introducing the cyclostationary feature of GPS signal, the GPS signal detection scheme based on cyclostatistics will be introduced.

Since the GPS signals adopt Binary Phase Shift Keying (BPSK) modulation method, the ideal GPS signal can be described below as:

$$
s(t)=\sum_{n=0}^{1022} q\left(t-n T_{c}-t_{0}\right) \cos \left(2 \pi f_{0} t+\theta_{n}\right)
$$

where:

$T_{c}$ denotes the time duration of one PRN code chip.

$q(t)$ is the square wave with duration $T_{c}$.

$t_{0}$ is the initial PRNcode phase.

$f_{0}$ is the signal carrier frequency.

$\theta_{n}$ is the carrier phase sequence which hops between 0 and $\pi$.

According to the work of Gardner (Gardner, 1994; Gardner et al., 2006 and Gardner, 1987), the theoretic cyclic spectrum of GPS signal (Dandawate and Giannakis, 1994) can be given in Equation (8). Figure 4 illustrates the cyclic spectrum of the GPS signal.

$$
S_{G P S}^{\alpha}(f)=\left\{\begin{array}{c}
\frac{1}{4 T_{c}} Q\left(f+\frac{\alpha}{2} \mp f_{0}\right) Q^{*}\left(f-\frac{\alpha}{2} \mp f_{0}\right) e^{-j\left[2 \pi\left(\alpha \mp 2 f_{0}\right) t_{0} \mp 2 \psi_{0}\right]}, \alpha= \pm 2 f_{0}+\frac{k}{T_{c}} \\
\frac{1}{4 T_{c}}\left[Q\left(f+\frac{\alpha}{2}+f_{0}\right) Q^{*}\left(f-\frac{\alpha}{2}+f_{0}\right)+Q\left(f+\frac{\alpha}{2}-f_{0}\right) Q^{*}\left(f-\frac{\alpha}{2}-f_{0}\right)\right] e^{-j 2 \pi \alpha t_{0}}, \\
\alpha=\frac{k}{T_{c}}
\end{array}\right.
$$

3.2. Cyclostationarity Feature of the GPS Signal under Multiplicative and Additive Noise. The cyclostationary feature of the GPS signal under the multiplicative and 

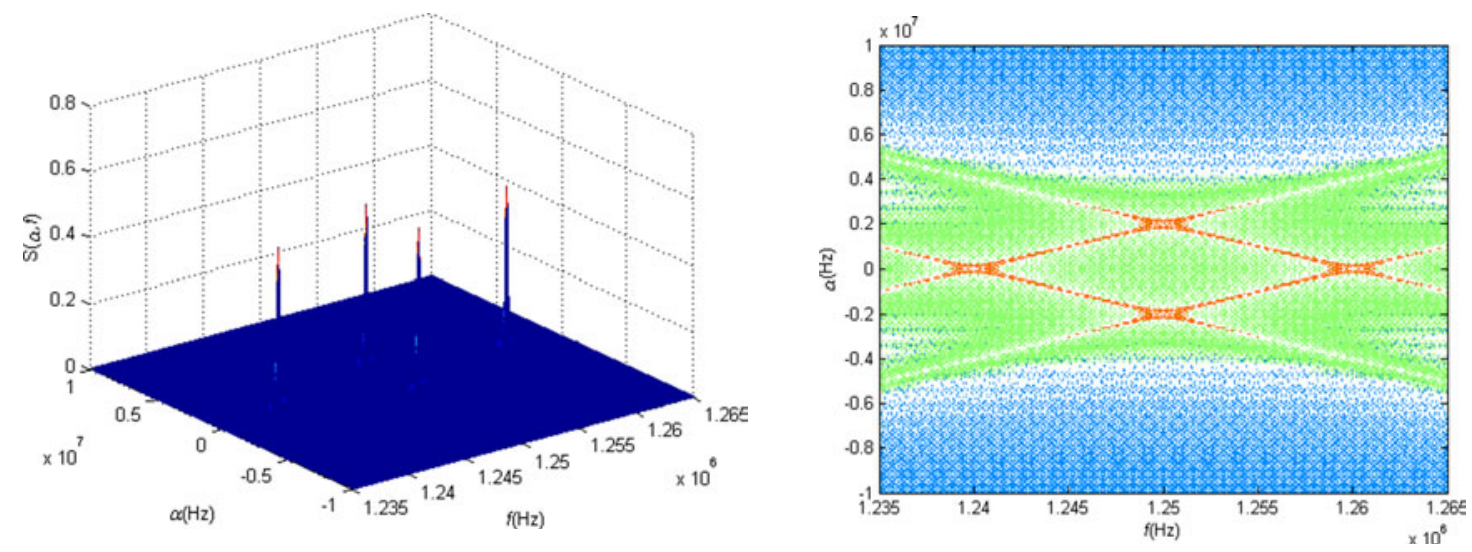

$J$
0
0
$z$
$a$
3
$z$
0
0
$\exists$
$\mathbb{7}$
0
0

(a) Three dimensional GPS signal cyclic-spectrum (b) Contour of GPS cyclic spectrum

Figure 4. Cyclic spectrum of the ideal GPS signal. 
GPS Sat. 2

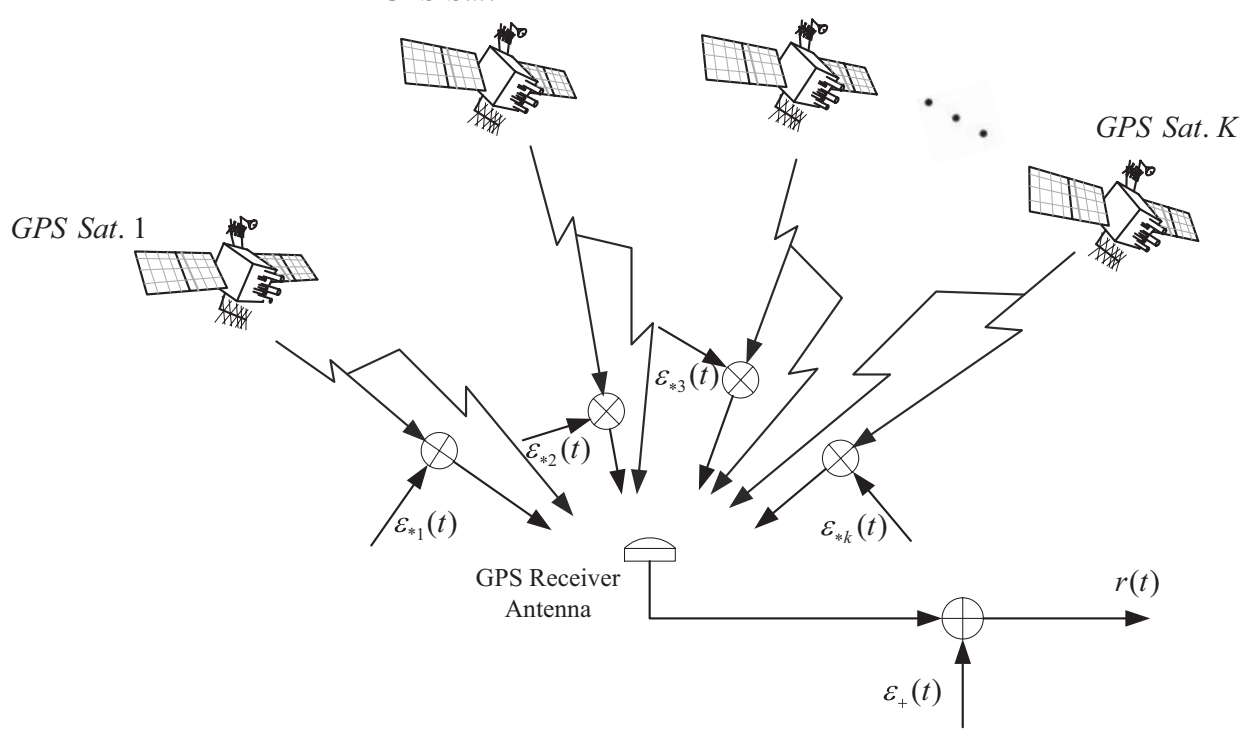

Figure 5. Multiplicative noise and the additive noise on the received GPS signal.

additive noise will be introduced in this subsection. Consider $K$ visible GPS satellites in sky as shown in Figure 5; i.e., number of satellites that have a direct line-of-sight path with the GPS receiver under investigation.

The GPS C/A-code waveform transmitted from a $k$ th GPS satellite, on the $L_{1}, L_{2}$, or $L_{5}$ frequencies, $x_{k}\left(t_{k}\right)$ is given by (Progri et al., 2006):

$$
x_{k}\left(t_{k}\right)=c_{k}\left(t_{k}\right) d_{k}\left(\left[t_{k}\right]_{D}\right) \cos \left(2 \pi L_{i} t_{k}+\theta_{k 0}\right)
$$

where:

$A_{k}$ is the amplitude of $k$ th GPS signal.

$t_{k}$ is the time of transmission of the $k$ th GPS signal.

$c_{k}\left(t_{k}\right)$ is PRN code at time $t_{k}$ which for the GPS $L_{1}, L_{2}$, or $L_{5}$ is the C/A code with code repetition sequence period at $1 \mathrm{~ms} ; L_{i}$ is carrier frequency.

$d_{k}\left(\left[t_{k}\right]_{D}\right)$ is the data bit transition for satellite $k$ spread by the code $c_{k}\left(t_{k}\right)$.

$D$ is the period when a data bit transition occurs (for the GPS L1 data case, it is equal to $20 \mathrm{~ms}$ ).

$\theta_{k 0}$ is some initial carrier phase of the signal; and the index $k=\{1 \ldots K\}$.

According to the GPS signal model presented in Figure 2, the received GPS signal simultaneously degraded by multiplicative noise and additive noise can be expressed at the discrete index, $n$, as in Equation (10).

$$
x(n)=\left\{\begin{array}{c}
\sum_{k=1}^{K} A_{k} c_{k}\left(n T_{s}-\tau_{k}\right) d_{k}\left([n]_{D}\right) \cos \left(2 \pi f_{k}\left(n T_{s}-\tau_{k}\right)\right)+ \\
\sum_{k=1}^{K} A_{k} c_{k}\left(n T_{s}-\tau_{k}\right) d_{k}\left([n]_{D}\right) \cos \left(2 \pi f_{k}\left(n T_{s}-\tau_{k}\right)\right) \varepsilon_{*}(n)+\varepsilon_{+}(n)
\end{array}\right.
$$


where:

$f_{k}$ denotes the $k$ th visible GPS satellite Doppler frequency.

$T_{s}$ is the sample period in seconds $(s)$.

$\tau_{s}$ is the residual code phase delay.

$\varepsilon *(n)$ denotes multiplicative noise process.

$\varepsilon_{+}(n)$ denotes additive Gaussian noise process.

The first part of Equation (10) represents the GPS signal model when multiplicative noise can be ignored.

The second part describes the GPS signal model when multiplicative noise is taken into consideration.

For the purpose of simplifying our analysis of the received GPS signal with the multiplicative and additive noise, in Equation (10), we consider the difference of the received GPS signal, $x(n)$, with Line Of Sight (LOS) GPS signal, $x_{L O S}(n)$, given by:

$$
\Delta x(n)=\left\{\begin{array}{c}
x(n)-x_{L O S}(n)= \\
\sum_{k=1}^{K} A_{k} c_{k}\left(n T_{s}-\tau_{k}\right) d_{k}\left([n]_{D}\right) \cos \left(2 \pi f_{k}\left(n T_{s}-\tau_{k}\right)\right) \varepsilon_{*}(n)+\varepsilon_{+}(n)
\end{array}\right.
$$

where:

$$
x_{L O S}(n)=\sum_{k=1}^{K} A_{k} c_{k}\left(n T_{s}-\tau_{k}\right) d_{k}\left([n]_{D}\right) \cos \left(2 \pi f_{k}\left(n T_{s}-\tau_{k}\right)\right)
$$

The first part of Equation (10), i.e., when the multiplicative noise can be ignored, is generalized into the special case that multiplicative noise equals to zero $(\varepsilon *(n)=0)$. Because we are only interested in the feasibility study of this multiplicative noise model and because the LOS effect has already been assessed in many publications it is therefore a lot easier to work with $\Delta x(n)$ instead of $x(n)$.

In order to acquire the GPS signal, correlation between the received GPS signal $\Delta x(n)$ and the locally generated identical replica $l(n)$ corresponding to the $k$ th GPS satellite signal, is performed; where, assuming that there is no Doppler effect on the locally generated signal we have:

$$
l(n)=c_{k}\left(n T_{s}\right)
$$

The expectation of $\Delta x(n)$ is zero as shown in the following:

$$
E[\Delta x(n)]=E\left[\sum_{k=1}^{K} A_{k} C_{k}\left(\tilde{\tau}_{k}\right) d_{k}\left[(n)_{D}\right] \cos \left(2 \pi f_{k} \tilde{\tau}_{k}\right) \varepsilon_{*}(n)+\varepsilon_{+}(n)\right]=0
$$

where $\tilde{\tau}_{k}=n T_{s}-\tau_{k}$.

The second-order statistics of $\Delta \mathrm{x}(\mathrm{n})$ are:

$$
E\left[\Delta x\left(n^{+}\right) \Delta x^{*}\left(n^{-}\right)\right]=E\left[\begin{array}{c}
\left\{\sum_{k=1}^{K} A_{k} c_{k}\left(\tilde{\tau}_{k}^{+}\right) d_{k}\left(\left[n^{+}\right]_{D}\right) \cos \left(2 \pi f_{k} \tilde{\tau}_{k}^{+}\right) \varepsilon_{*}\left(n^{+}\right)+\varepsilon_{+}\left(n^{+}\right)\right\} \\
\left\{\sum_{k^{\prime}=1}^{K} A_{k^{\prime}} c_{k^{\prime}}\left(\tilde{\tau}_{k^{\prime}}^{-}\right) d_{k^{\prime}}\left(\left[n^{-}\right]_{D}\right) \cos \left(2 \pi f_{k^{\prime}} \tilde{\tau}_{k^{\prime}}^{+}\right) \varepsilon_{*}\left(n^{-}\right)\right\}
\end{array}\right]
$$


where:

$$
\begin{aligned}
\tilde{\tau}_{k}^{+} & =n^{+} T_{s}-\tau_{k}, \tilde{\tau}_{k^{\prime}}^{-}=n^{-} T_{s}-\tau_{k^{\prime}} . \\
n^{+} & =n+\frac{m}{2} . \\
n^{-} & =n-\frac{m}{2} .
\end{aligned}
$$

Due to independence between multiplicative noise $\varepsilon_{*}(n)$ and additive noise $\varepsilon_{+}(n)$, and the means of $\varepsilon *(n)$ and noise $\varepsilon_{+}(n)$ are zero; therefore, Equation (15) can be rewritten as:

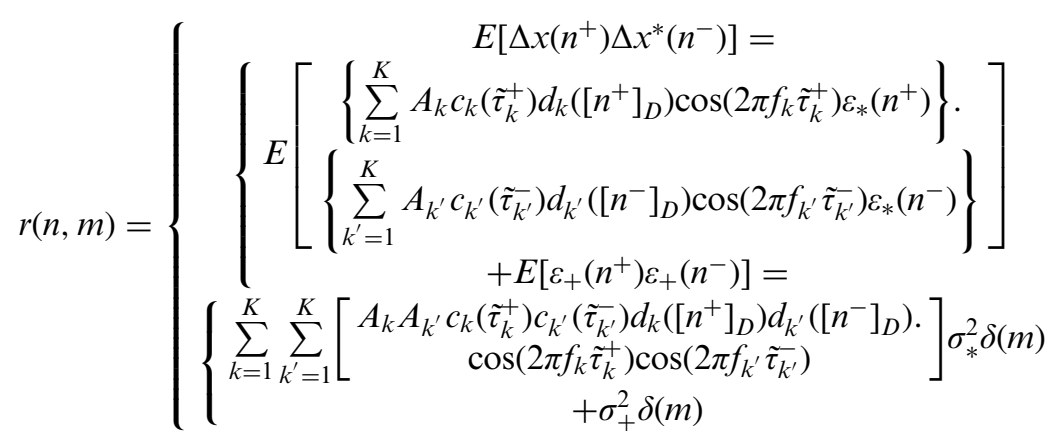

Because of the orthogonality of the pseudo random code $C_{k}(n)$ in GPS signal, $r(n, m)$ has a none-zero contribution item when $k=k^{\prime}$. Therefore, Equation (16) can be rewritten as:

$$
r(n, m)=\left\{\begin{array}{c}
\sum_{k=1}^{K} A_{k}^{2} c_{k}\left(\tilde{\tau}_{k}^{+}\right) c_{k^{\prime}}\left(\tilde{\tau}_{k^{\prime}}\right) d_{k}\left(\left[n^{+}\right]_{D}\right) d_{k^{\prime}}\left(\left[n^{-}\right]_{D}\right) \\
\cos \left(2 \pi f_{k} \tilde{\tau}_{k}^{+}\right) \cos \left(2 \pi f_{k} \tilde{\tau}_{k^{\prime}}^{-}\right) \sigma_{*}^{2} \delta(m)
\end{array}\right.
$$

From Equation (8), the GPS signal under additive and multiplicative noise is not wide sense stationary, because the second order statistics $r(n, m)$ of received signal $\Delta x(n)$ relate to the initial time $n T_{s}$.

When multiplicative noise is extremely low, the first part in the result of Equation (17) can be ignored. In such a condition, GPS signal under multiplicative and additive noise can be considered to be wide sense stationary. That is a reason why the traditional method based on GPS signal correlation can successfully acquire the GPS signal. However, if the multiplicative noise is large enough, the correlation result is related to time $n T_{s}$. Therefore, under the condition of large multiplicative noise, the noise processes of the received GPS signal cannot be modelled as wide sense stationary and the conventional GPS signal acquisition method based on correlation may not always work.

Fortunately, the second order statistics $r(n, m)$ of GPS signal $\Delta x(n)$ is periodic versus time $n T_{s}$. Furthermore, there are two periods for the correlation function $r(n, m)$, the period of PRN code $c_{k}(n)$ which is $1 \mathrm{~ms}$, and the period of the carrier frequency which is $T_{c}=1 / f_{c}$. Since of the two periods, the period of GPS signal $\Delta x(n)$ should be the lowest common multiple of $1 \mathrm{~ms}$ and $T_{c}$. Indeed, the carrier frequency period might differ from the different local oscillator frequencies, and different Doppler shifts also change the carrier frequency. Therefore, getting the lowest common multiple of $1 \mathrm{~ms}$ and $T_{c}$ is not a good choice to achieve GPS signal acquisition under the multiplicative 

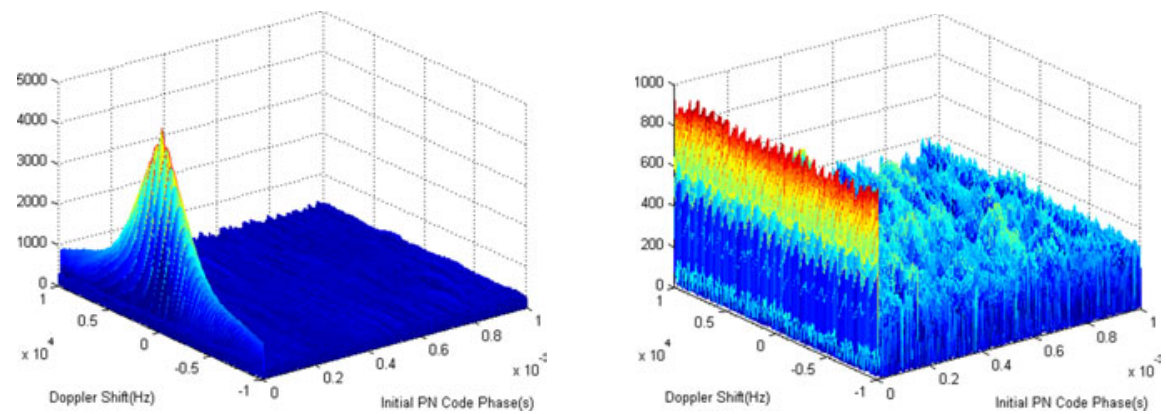

Correlation results (a) without compensation to Doppler shift caused waveform distortion (CDSCWD) and (b) with CDSCWD

Figure 6. Contrast before and after GPS signal waveform distortion compensations.

and additive noise. For this reason another strategy is preferred, which is to eliminate one of the two periods from the periodicity of correlation function, $r(n, m)$. Since the period of PRN code is relatively more fixed than the period of the carrier frequency, the PRN code should be tentatively eliminated before achieving successful GPS signal acquisition.

\section{GPS SIGNAL ACQUISITION SCHEME UNDER MULTI- PLICATIVE AND ADDITIVE NOISE.}

4.1. Compensation to Waveform Distortion by Doppler Shift. Doppler shift not only changes the carrier frequency, but also distorts the wave form of the GPS signal. Psiaki (2001) presents the distortion effect on the received waveform of the GPS signal by Doppler shift, which is depicted as:

$$
c[(1+\eta) t]
$$

where:

$\eta$ is the distortion factor

$\eta=f_{d} / 1.575 \times 10^{6}[\mathrm{kHz}]$

$f_{d}$ denotes Doppler shift frequency in [kHz].

Based on the waveform distortion formula Equation (18), the $10 \mathrm{kHz}$ Doppler shift on the GPS signal with $1 \mathrm{~ms}$ causes an extension to the GPS code length by about seven chips; such a seven chips length shift represents a disaster to the GPS signal acquisition. To guarantee stable performance on GPS signal acquisition, the maximum length change should be controlled in the range of a half-chip. Similarly, the maximum Doppler shift allowed is about $787 \cdot 5 \mathrm{~Hz}$ based on Equation (18); therefore, a block of GPS signal local replica is needed to decrease the possible Doppler shift. To guarantee the maximum Doppler shift of $787 \cdot 5 \mathrm{~Hz}$, twenty six elements of the block, which have the same frequency interval, are needed to compensate the Doppler-caused waveform distortion. The compensation effectiveness is demonstrated in Figure 6b; in contrast, the correlation result without compensation is presented in Figure 6a.

In Figure 6a, if there is no compensation to the Doppler shift distortion, the correlation peak value decreases sharply with the increase of the Doppler shift. 
In Figure 6b, the correlation peak value between the received GPS signal and the local replica keeps quite stable and it can be easily distinguished from the noise background. Therefore, the compensation to the waveform distortion caused by Doppler shift is good method.

4.2. GPS Signal Detection under Multiplicative and Additive Noise. After eliminating the effect on second-order statistics of GPS signal caused by PRN code periodicity, we calculate the second-order statistics between received GPS data and local replica of GPS signal from one satellite. Furthermore, for the simplicity of our analysis, consider the case with received GPS signal from only one satellite. Therefore, Equation (9) can be rewritten as:

$$
x^{\prime}(n)=A_{k} \cos \left(2 \pi f_{c} \tilde{\tau}_{0}\right) \varepsilon_{*}(n)+\varepsilon_{+}(n)
$$

Such a simplification is reasonable because when detecting a desired GPS signal, the locally generated PRN code replica is multiplied with the received GPS signal, which eliminates the effect caused by pseudo random sequence in the received GPS signal. Simultaneously, the effect of the PRN sequence from other GPS satellites is also eliminated. Afterwards, $r(n, m)$ can be rewritten as:

$$
r_{x^{\prime}}(n, m)=\left\{\begin{array}{c}
A_{k}^{2} \cos \left(2 \pi f_{c} \hat{\tau}_{0}\right) \cos \left(-2 \pi f_{c} \breve{\tau}_{0}\right) \sigma_{*}^{2} \delta(m)+\sigma_{+}^{2} \delta(m)= \\
\frac{1}{2} A_{k}^{2}\left[\cos \left(2 \pi f_{c} m T_{s}\right)+\cos \left(2 \pi f_{c} 2 n T_{s}-\theta_{0}\right)\right] \sigma_{*}^{2} \delta(m)+\sigma_{+}^{2} \delta(m)
\end{array}\right.
$$

where:

$$
\begin{aligned}
& \hat{\tau}_{0}=\left(n+\frac{m}{2}\right) T_{s}-\tau_{0} . \\
& \breve{\tau}_{0}=\left(n-\frac{m}{2}\right) T_{s}-\tau_{0}, \\
& \theta_{0}=2 \pi f_{c} \tau_{0} .
\end{aligned}
$$

For the zero-lag autocorrelation $(m=0), r_{x^{\prime}}(n, m)$ is rewritten as:

$$
r_{x^{\prime}}(n, 0)=\frac{1}{2} A_{k}^{2} \cos \left(2 \pi f_{c} 2 n T_{s}-\theta_{0}\right) \sigma_{*}^{2}+\frac{1}{2} A_{k}^{2} \sigma_{*}^{2}+\sigma_{+}^{2}
$$

From Equation (20), the zero-lag second-order statistics $r_{x^{\prime}}(n, 0)$ of $x^{\prime}(n)$ are periodic with $T=1 / 2 f_{c}$, and the expectation of $x^{\prime}(n)$ is zero. Therefore, the received GPS signal is cyclostationary. Therefore, the carrier frequency of $x^{\prime}(n)$ can be estimated; after that, the Doppler shift of the GPS signal can be calculated as:

$$
\hat{f}_{d}=\hat{f}_{c}-f_{0}
$$

where:

$\hat{f}_{c}$ is the estimated carrier frequency.

$f_{0}$ is the ideal carrier frequency.

The initial PRN code phase is determined by the tentative test of getting rid of the PRN sequence effect. Once Doppler shift and initial code phase are determined, the GPS receiver can successfully perform the GPS signal tracking. 
The correlation function $r_{x^{\prime}}(n, 0)$ can be expanded in a Fourier series as (Gardner, 1991):

$$
r_{x^{\prime}}(n, 0)=\frac{1}{L} \sum_{l=0}^{L-1} R_{x^{\prime}}^{l \alpha_{0}} e^{j 2 \pi l \alpha_{0} n T_{s}}
$$

where:

$\alpha=l \alpha_{0}$ is cyclic frequency.

$\alpha_{0}$ is the basic cyclic frequency which equals to $2 f_{c}$.

The Fourier series at cyclic frequency $\alpha$ can be calculated as:

$$
R_{x^{\prime}}^{\alpha}=\left\{\begin{array}{c}
\left(r_{x^{\prime}}(n, 0) e^{-j 2 \pi n T_{s}}\right)_{n}= \\
\frac{1}{2} A_{k}^{2} \cos \left(2 \pi f_{c} 2 n T_{s}-\theta_{0}\right) \sigma_{*}^{2}+\frac{1}{2} A_{k}^{2} \sigma_{*}^{2}+\sigma_{+}^{2}= \\
\frac{1}{4} A_{k}^{2} \sigma_{*}^{2}\left[e^{-j \theta_{0}} \delta\left(\alpha+2 f_{c}\right)+e^{j \theta_{0}} \delta\left(\alpha-2 f_{c}\right)\right]+\left(\frac{1}{2} A_{k}^{2} \sigma_{*}^{2}+\sigma_{+}^{2}\right) \delta(\alpha)
\end{array}\right.
$$

From Equation (24), there are several special cyclic frequency points. The first cyclic frequency point at $\alpha=0$ is given by:

$$
R_{x^{\prime}}^{\alpha}=\frac{1}{2} A_{k}^{2} \sigma_{*}^{2}+\sigma_{+}^{2}
$$

The second cyclic frequency point at $\alpha=2 f_{c}$ is given by:

$$
R_{x^{\prime}}^{\alpha}=\frac{1}{4} A_{k}^{2} \sigma_{*}^{2} e^{j \theta_{0}}
$$

The third cyclic frequency point at $\alpha=-2 f_{c}$ is given by:

$$
R_{x^{\prime}}^{\alpha}=\frac{1}{4} A_{k}^{2} \sigma_{*}^{2} e^{-j \theta_{0}}
$$

For any other cyclic frequency points, $\left\{\forall \alpha \mid \alpha \neq 0,2 f_{c},-2 f_{c}\right\}$

$$
R_{x^{\prime}}^{\alpha}=0
$$

From the analysis above, there are peak values of Fourier series for the zero-lag second order cyclic statistics at cyclic frequencies $\alpha=\left\{0,2 f_{c},-2 f_{c}\right\}$. Furthermore, at the cyclic frequency $\alpha=0$, the Fourier series of GPS signal second order cyclic statistics is degraded by additive Gaussian noise. In the condition of low signal to noise ratio, GPS signal will submerge in the additive noise. Therefore, the cyclic frequencies at $\alpha= \pm 2 f_{c}$ are preferred to implement the GPS signal detection. To estimate the carrier frequency, the cyclic spectrum at cyclic frequency point $\alpha=2 f_{c}$ or $\alpha=-2 f_{c}$ is calculated. Afterwards, the peak detection is performed to determine whether the de-spreaded GPS signal is detected.

4.3. GPS Signal Detection Block Diagram. After introducing the principle of detecting the GPS signal with multiplicative and additive noise, GPS signal acquisition method based on cyclo-stationarity is given in this subsection.

To demonstrate the GPS detection method, Figure 7 presents the GPS signal detection block diagram. As depicted in Figure 7, multiply the received GPS signal $x(n)$ with local replica bit by bit. The bit multiplication aims at eliminating PRN code 


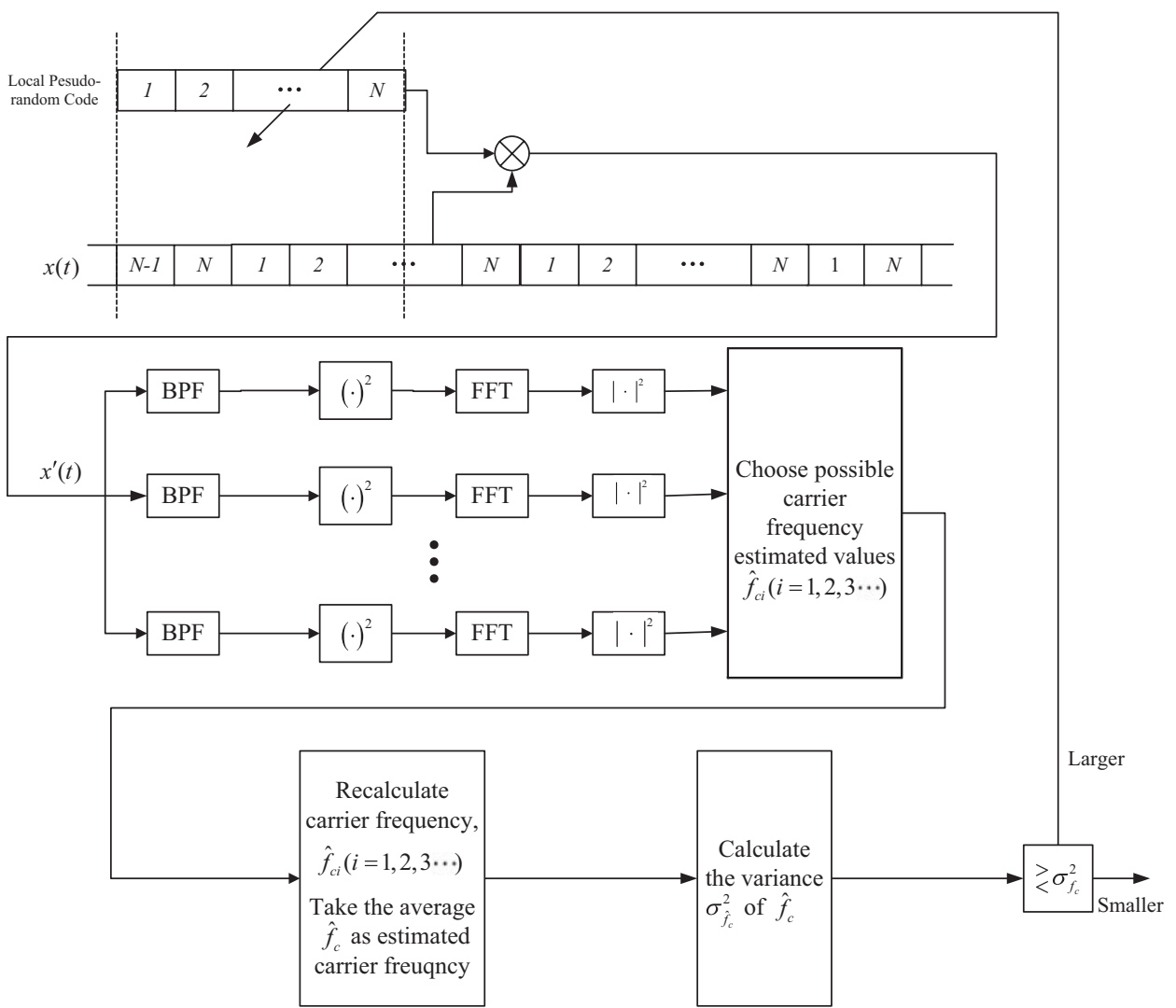

Figure 7. A generic GPS signal detection block diagram.

tentatively and getting rid of the disturbance caused by two periods in the cyclostationarity of the GPS signal. After the bit multiplication, pose the output $x^{\prime}(n)$ into the GPS signal detection module based on cyclostationarity. In the detection module, calculate the periodic second order statistic with the zero-lag $\hat{r}_{x}(n, 0)$; then compute the coefficient of Fourier series $\hat{R}_{x}^{\alpha}$ versus the cyclic frequency $\alpha$; afterwards, search the peak absolute value of the Fourier series $\hat{R}_{x}^{\alpha}$ over the cyclic frequency $\alpha$; after the peak absolute value is fixed, the estimated carrier frequency is achieved with one half of the cyclic frequency corresponding to the peak absolute value $\left\{\hat{f}_{c}=0.5 \alpha \mid \max \left(\hat{R}_{x}^{\alpha}\right)\right\}$; to guarantee the accuracy of the estimated carrier frequency, multiple iterations of the estimation can be performed and take the average as the final estimated value.

\section{AMBIGUITY FUNCTION OF THE GPS SIGNAL DETECTION} UNDER MULTIPLICATIVE AND ADDITIVE NOISE. In the last section, the Doppler shift and the initial PRN code phase estimation methods were presented. In this section, we pay attention to the accuracy of the estimated values. The ambiguity functions of the estimated Doppler shift and the initial code phase are analysed which reflect the accuracy of the estimated values.

The deduction on the equations above corresponds to the ideal condition which is not identical with engineering practice. For example, Equation (4) contains an item of 
the impulse function, $\delta(\cdot)$, which is a mathematical representation of the autocorrelation function of an ideal additive Gaussian noise process. In this article, triangular function, $\Lambda(m)$, is utilized as an approximation of the delta impulse function, $\delta(\cdot)$. Suppose that the GPS signal window width used for the autocorrelation calculation is $\Delta \tau$. The autocorrelation function at the delay $m T_{s}$ is written as:

$$
\hat{r}_{x^{\prime}}(n, m)=\frac{1}{2} A_{k}^{2}\left[\cos \left(2 \pi f_{c} m T_{s}\right)+\cos \left(2 \pi f_{c} 2 n T_{s}-\theta_{0}\right)\right] \sigma_{*}^{2} \Lambda_{\Delta \tau}(m)+\sigma_{+}^{2} \Lambda_{\Delta \tau}(m)
$$

where:

$$
\begin{aligned}
& \Lambda_{\Delta \tau}(m)=\left\{\begin{array}{cc}
-\left|m M^{2} T_{s}^{2}\right|+M T_{s}, & \left|m T_{s}\right| \leq 1 / \Delta \tau \\
0, & \text { elsewhere }
\end{array}\right. \\
& \mathbf{M}=\left[\Delta \tau / T_{s}\right] .
\end{aligned}
$$

Furthermore:

$$
\hat{r}_{x^{\prime}}(n, m)=\sum_{l=-\infty}^{+\infty} \hat{r}_{x^{\prime}} \alpha_{0}(m) e^{j 2 \pi l \alpha_{0} T_{s}}
$$

With duration $\Delta \tau$ of the processed GPS signal, the Fourier series of the periodic second order statistics $\hat{r}_{x^{\prime}}(n, m)$ at cyclic frequency $\alpha$ is given by:

$$
\hat{r}_{x^{\prime}}(n, m)=\frac{1}{L} \sum_{l=0}^{L-1} \hat{R}_{x^{\prime}}^{l \alpha_{0}} e^{j 2 \pi l \alpha_{0} T_{s}}
$$

and:

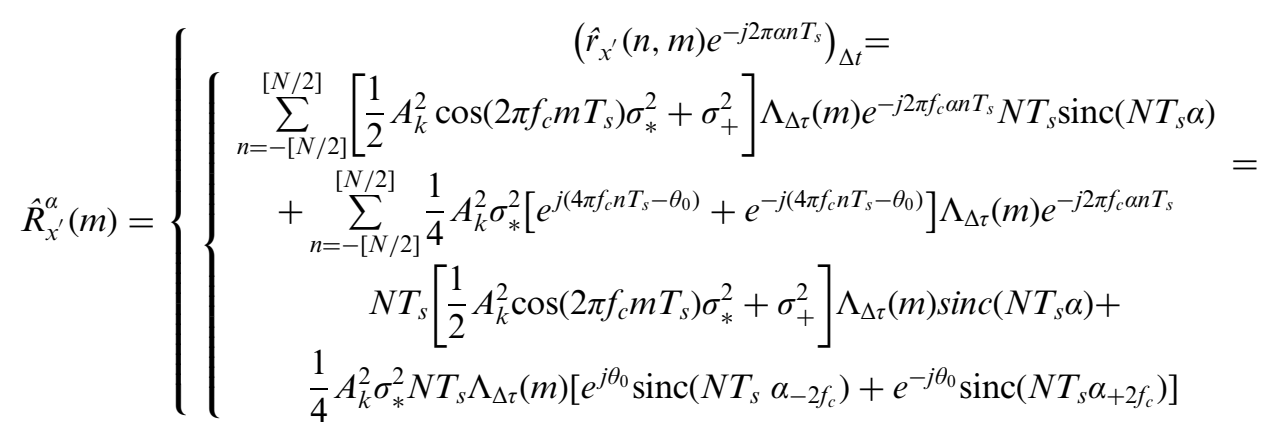

where:

$$
\begin{aligned}
& \alpha_{-2 f_{c}}=\alpha-2 f_{c}, \\
& \alpha_{+2 f_{c}}=\alpha+2 f_{c}, \\
& N=\Delta \tau / T_{s} .
\end{aligned}
$$

From Equation (32), to distinguish two possible peak value of $\hat{r}_{x^{\prime}}^{\alpha}(M)$, the inequality below Equation (33) should be guaranteed:

$$
4 f_{c} \gg \frac{1}{N T_{s}} \text { or } 4 f_{c} N T_{s} \gg 1
$$


Furthermore, at the different cyclic frequencies, the cyclo-stationarity of the GPS signal can be calculated as follows. At cyclic frequency point $\alpha=0$ is given by:

$$
\hat{R}_{x^{\prime}}^{\alpha}(m)=\left\{\begin{array}{c}
N T_{s}\left[\frac{1}{2} A_{k}^{2} \cos \left(2 \pi f_{c} m T_{s}\right) \sigma_{*}^{2}+\sigma_{+}^{2}\right] \Lambda_{\Delta \tau}(m)+ \\
\frac{1}{4} A_{k}^{2} \sigma_{*}^{2} N T_{s} \Lambda_{\Delta \tau}(m)\left[e^{j \theta_{0}} \operatorname{sinc}\left(-2 N T_{s} f_{c}\right)+e^{-j \theta_{0}} \operatorname{sinc}\left(2 N T_{s} f_{c}\right)\right]
\end{array}\right.
$$

However, under the condition of $4 f_{c} N T_{s} \gg 1$, the factor $\operatorname{sinc}\left(2 N T_{s} f_{c}\right)$ induces extremely huge losses and $\hat{R}_{x^{\prime}}^{\alpha}(m)$ can be deduced as:

$$
\hat{R}_{x^{\prime}}^{\alpha}(m) \cong N T_{s}\left[\frac{1}{2} A_{k}^{2} \cos \left(2 \pi f_{c} m T_{s}\right) \sigma_{*}^{2}+\sigma_{+}^{2}\right] \Lambda_{\Delta \tau}(m)
$$

At cyclic frequency point $\alpha=2 f_{c}$ is given by:

$$
\hat{R}_{x^{\prime}}^{\alpha}(m)=\left\{\begin{array}{c}
N T_{s}\left[\frac{1}{2} A_{k}^{2} \cos \left(2 \pi f_{c} m T_{s}\right) \sigma_{*}^{2}+\sigma_{+}^{2}\right] \Lambda_{\Delta \tau}(m) \operatorname{sinc}\left(2 N T_{s} f_{c}\right)+ \\
\frac{1}{4} A_{k}^{2} \sigma_{*}^{2} N T_{s} \Lambda_{\Delta \tau}(m)\left[e^{j \theta_{0}}+e^{-j \theta_{0}} \operatorname{sinc}\left(4 N T_{s} f_{c}\right)\right]
\end{array}\right.
$$

Similarly, under the condition of $4 f_{c} N T_{s} \gg 1$, both $\operatorname{sinc}\left(2 N T_{s} f_{c}\right)$ and $\operatorname{sinc}\left(4 N T_{s} f_{c}\right)$ will induce huge losses, therefore $\hat{R}_{x^{\prime}}^{\alpha}(m)$ can be proximately written as:

$$
\hat{R}_{x^{\prime}}^{\alpha}(m) \cong \frac{1}{4} A_{k}^{2} \sigma_{*}^{2} N T_{s} \Lambda_{\Delta \tau}(m) e^{j \theta_{0}}
$$

At cyclic frequency point $\alpha=-2 f_{c}$, the similar conclusion of Equation (37) can be written as:

$$
\hat{R}_{x^{\prime}}^{\alpha}(m) \cong \frac{1}{4} A_{k}^{2} \sigma_{*}^{2} N T_{s} \Lambda_{\Delta \tau}(m) e^{-j \theta_{0}}
$$

First, perform Fourier transformation on $\hat{R}_{x^{\prime}}^{\alpha}(m)$ at the different cyclic frequencies; second, the realistic cyclic spectrum of $x^{\prime}(n)$ is obtained. At cyclic frequency point $\alpha=0$ is given by:

$$
\Delta \hat{S}_{x^{\prime}}^{\alpha}(f) \cong\left[\frac{1}{2 N T_{s}} A_{k}^{2} \cos \left(2 \pi f_{c} m T_{s}\right) \sigma_{*}^{2}+\sigma_{+}^{2}\right] \operatorname{sinc}^{2}\left(f / N T_{s}\right)
$$

At cyclic frequency point $\alpha= \pm 2 f_{c}$ is given by:

$$
\hat{S}_{x^{\prime}}^{\alpha}(f) \cong \frac{1}{4 N T_{s}} A_{k}^{2} \sigma_{*}^{2} \operatorname{sinc}^{2}\left(f / N T_{s}\right) e^{j \theta_{0}}
$$

To avoid the degradation of the additive noise, the cyclic spectrum at cyclic frequency $\alpha= \pm 2 f_{c}$ is preferred to detect the GPS signal, rather than the cyclic spectrum at cyclic frequency $\alpha=0$.

6. SIMULATION AND EXPERIMENT. To test the effectiveness of the proposed GPS signal detection scheme, simulations and experiments are performed in this section. In the first part of the simulations, the carrier frequency estimation is performed under different multiplicative noise levels. The received GPS signal is acquired on a street located in the downtown area of a city with a high density of 
buildings. The different SNR of the GPS signal is implemented with the help of an attenuator.

To the best knowledge of the author, there is no common SNR definition for the GPS signal under multiplicative and additive noise. Referring to the work by Ghogho et al., (1998) and considering the simulation results in Figure 3e, the SNR definition is given as:

$$
S N R_{M A}=\frac{A_{s}^{2}}{\frac{A^{2}}{A_{0}^{2}} \cdot B \cdot\left(\frac{\sigma_{*}^{2}}{\sigma_{* 0}^{2}}-1\right) \sigma_{* 0}^{2}+B \cdot \sigma_{+}^{2}}
$$

where:

$A_{s}^{2}$ denotes the signal power.

$A$ denotes the amplitude of the GPS signal.

$A_{0}$ denotes the GPS signal baseline which is set at $10^{-9} m v^{2}$.

$B$ is the bandwidth of the GPS signal.

The subscript MA indicates the SNR is in the condition with Multiplicative and Additive (MA) noise.

The variable $\sigma_{*_{0}}^{2}$ is the reference value of the multiplicative noise strength beyond which the multiplicative noise needs to be taken into consideration; otherwise, the multiplicative noise can be ignored because the degradation by multiplicative noise is extremely slight. Referring the simulation result listed in Figure $3 \mathrm{e}$ the reference standard of multiplicative noise strength is $10^{-18 \cdot 5} \mathrm{mv}^{2}$, which value is selected due to the minute effect on the GPS signal acquisition.

From the definition of SNR under multiplicative and additive noise, it will regress to the common form $\left(S N R=A_{S}^{2} / \sigma_{+}^{2}\right)$ under additive noise when the multiplicative noise level equals the reference value $\left(\sigma_{*}^{2}=\sigma_{*}^{2}\right)$. The bandwidth B in the first item of the denominator is of no physical sense which is used to balance the effect of the additive noise and to reflect the effect of the multiplicative noise in the SNR definition.

Figure 8a illustrates the carrier frequency estimation with the GPS signal strength at $-160 \mathrm{dBw}=-190 \mathrm{dBm}$ (Anon, 2003), the multiplicative noise at $-165 \mathrm{dBm} / \mathrm{Hz}$ $\left(10 \log \sigma_{*}^{2}=-165 \mathrm{dBm} / \mathrm{Hz}\right)$ and the additive noise at $-174 \mathrm{dBm} / \mathrm{Hz}\left(10 \log \sigma_{+}^{2}=\right.$ $-174 \mathrm{dBm} / \mathrm{Hz}$ ); Figure $8 \mathrm{~b}$ depicts the carrier frequency estimation results in the conditions $10 \log A_{s}^{2}=-160 \mathrm{dBm} / \mathrm{Hz}, 10 \log \sigma_{*}^{2}=-170 \mathrm{dBm} / \mathrm{Hz}$ and $10 \log \sigma_{+}^{2}=$ $-174 \mathrm{dBm} / \mathrm{Hz}$; and Figure $8 \mathrm{c}$ represents the carrier frequency estimation results in the conditions, $10 \log \sigma_{*}^{2}=-174 \mathrm{dBm} / \mathrm{Hz}$ and $10 \log \sigma_{+}^{2}=-174 \mathrm{dBm} / \mathrm{Hz}$. The data length of the received GPS signal is $20 \mathrm{~ms}$, and the sampling frequency $f_{s}$ is $4 f_{0}$.

As shown in Figures $8 \mathrm{a}, 8 \mathrm{~b}$, and $8 \mathrm{c}$, the carrier frequency cannot be estimated with multiplicative noise at $10 \log \sigma_{*}^{2}=-165 \mathrm{dBm} / \mathrm{Hz}$; successful detection can be achieved with $-170 \mathrm{dBm} / \mathrm{Hz}$ multiplicative noise, but the variance of the estimated value is large. Under the multiplicative noise at $10 \log \sigma_{*}^{2}=-174 \mathrm{dBm} / \mathrm{Hz}$, the carrier frequency can be estimated with a small variance. Furthermore, to analyse the effectiveness of GPS signal detection, the effects on the variance from versatile factors are considered, such as multiplicative noise level, data block duration and the sampling frequency.

Figure $8 \mathrm{~d}$ illustrates the carrier frequency variance curve versus the multiplicative noise. The sampling frequency $f_{s}$ is $4 f_{0}$ and 2000 sampled points of the received data 


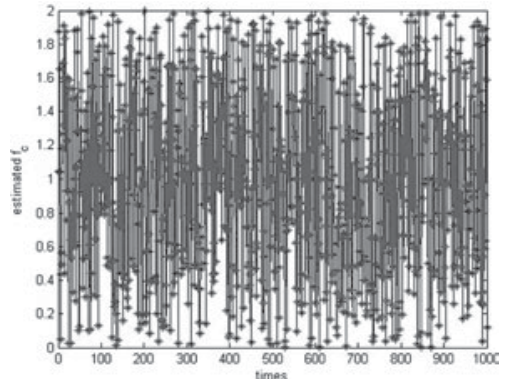

(a) $-165 \mathrm{dBm} / \mathrm{Hz}$ (heavy $\mathrm{MNE}^{1}$ )

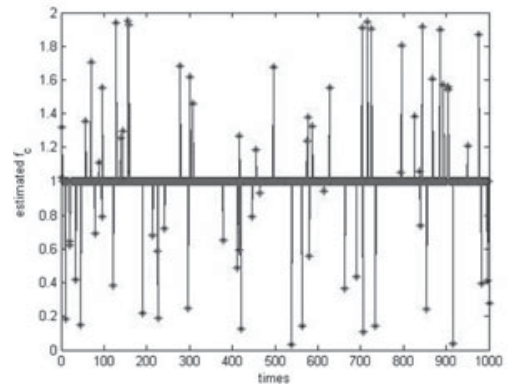

(c) $174 \mathrm{dBm} / \mathrm{Hz}$ (light MNE)

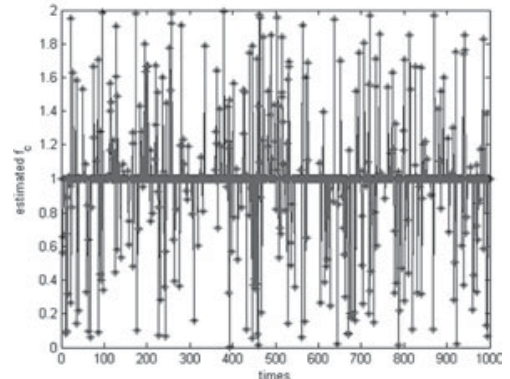

(b) $-170 \mathrm{dBm} / \mathrm{Hz}$ (average MNE)

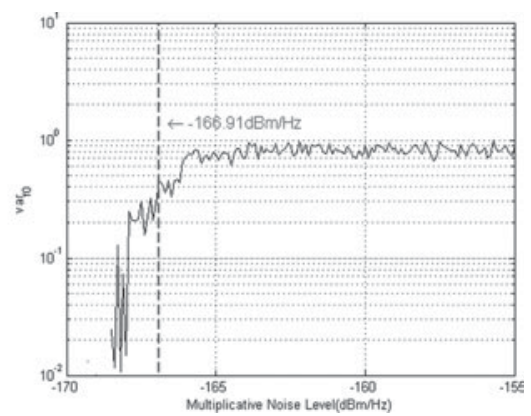

(d) Variance curve of estimated carrier frequency versus multi. noise power level

Figure 8. Carrier frequency estimation and variance result at different noise levels. ${ }^{1}$

are used. At each multiplicative noise power level, simulations are repeated 1000 times to calculate the variance. As shown in Figure 8d, there is a dashed vertical line at $-166.91 \mathrm{dBm} / \mathrm{Hz}$. On the right side of the dashed line, the variance of the estimated frequency is regarded to be too large to determine the carrier frequency.

The left side from the dashed line means the zone in which the carrier frequency can be estimated effectively. In this sense the dividing line at $-166.91 \mathrm{dBm} / \mathrm{Hz}$ multiplicative noise power level taken as the threshold indicates the successful carrier frequency estimation zone.

To illustrate the impact of sampling frequency on the carrier frequency estimation variance, simulations under different sampling frequencies were carried out. Three carrier frequency estimation variance curves are plotted in Figure 9, which corresponds to $4 f_{0}, 12 f_{0}$ and $20 f_{0}$ respectively. In the experiment, 2000 sampled points are used.

From Figure 9a, a high sampling frequency generates a better carrier frequency variance curve. Similarly, there are three dashed vertical lines which divide the zones of successful carrier frequency estimation zone and the unsuccessful zone. The vertical dashed lines are drawn at the multiplicative noise levels $-173.21 \mathrm{dBm} / \mathrm{Hz}$, $-171.51 \mathrm{dBm} / \mathrm{Hz},-170 \cdot 70 \mathrm{dBm} / \mathrm{Hz}$ respectively. The left side of the dashed line corresponds to the unsuccessful carrier frequency estimation zone. Clearly, the variance corresponding to the sampling frequency $4 f_{0}$ arrives at 0.3 at about

\footnotetext{
1 MNE: Multiplicative Noise Environment.
} 


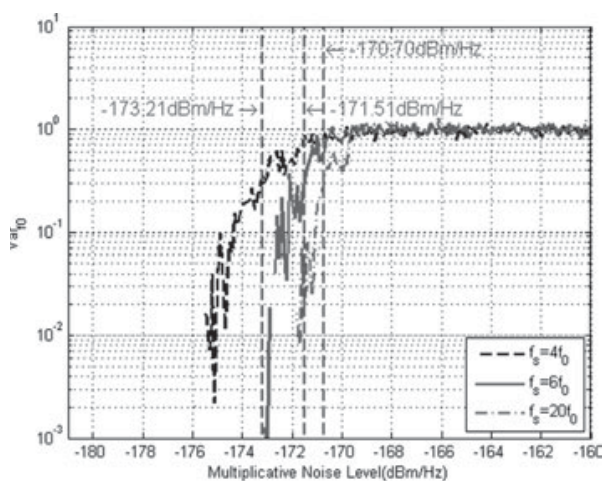

(a) sampling frequency

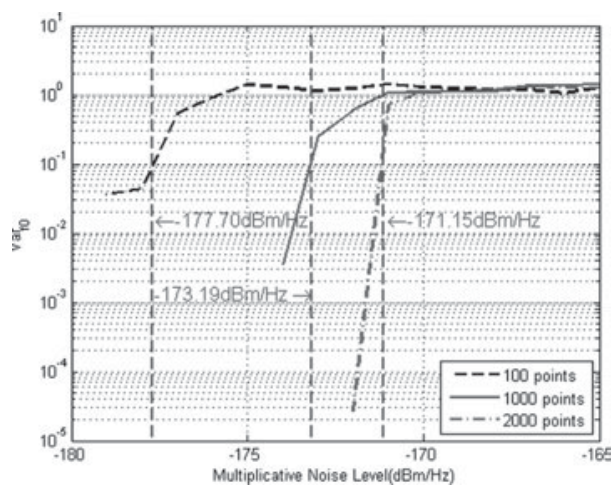

(b) received GPS signal duration.

Figure 9. Impact on variance curve.

$-173 \cdot 21 \mathrm{dBm} / \mathrm{Hz}$, while variance corresponding to sampling frequency $20 f_{0}$ needs the signal power at about $-170 \cdot 70 \mathrm{dBm} / \mathrm{Hz}$. The advantage of a high sampling frequency is caused by less information loss when performing analogue-to-digital transformation.

To illustrate the effect of different processed data block samples (or sample interval length), simulations were also carried out and the simulation results are presented in Figure $9 \mathrm{~b}$. Under the same sampling frequency $\left(10 f_{0}\right)$, the three estimated carrier frequency variance curves correspond to 100-point, 1000-point and 2000-point sample block intervals.

There are also three dashed vertical lines dividing the decision zones corresponding at the multiplicative noise levels $-177.70 \mathrm{dBm} / \mathrm{Hz},-173.19 \mathrm{dBm} / \mathrm{Hz}$ and $-171 \cdot 15 \mathrm{dBm} / \mathrm{Hz}$ respectively. From Figure $9 \mathrm{~b}$ in the condition of 100 sampled points, when the multiplicative noise level goes beyond $-177 \cdot 70 \mathrm{dBm} / \mathrm{Hz}$, the carrier frequency estimation variance is smaller than $0 \cdot 1$; in the condition of 1000 sampled points, the multiplicative noise level line is at about $-173 \cdot 19 \mathrm{dBm} / \mathrm{Hz}$; for 2000 sampled points, the multiplicative noise level is at about $-171 \cdot 15 \mathrm{dBm} / \mathrm{Hz}$. Therefore, the tendency is clear that the accuracy improves with number of samples in the block data interval.

7. CONCLUSIONS. This paper explains the multiplicative noise effects in the received GPS data. An effective GPS signal acquisition method is proposed which is tested by simulations and experiments. The existing weak GPS signal acquisition methods were summarized, which only considered the additive noise and neglected the degradation effects from the multiplicative noise. After that, the factors inducing multiplicative noise in the GPS signal were summarized, with the fast carrier phase transition due to electron and high density multipath.

To acquire the weak GPS signal under additive and multiplicative noise, the cyclostationarity of the GPS signal is utilized. The GPS signal possesses a cyclostationary feature under the two kinds of noise processes which can distinguish the GPS signal from the noise and interference. To implement the cyclostationarity based on the weak GPS signal acquisition method, the PRN code is tentatively 
removed. After that the period of the carrier is estimated by utilizing the cyclostationarity properties.

Finally, simulations and experiments are carried out to test the effectiveness of the proposed method and analyse the factors which affect the GPS signal acquisition performance. The experimental results show that the method can acquire extremely weak GPS signals under multiplicative and additive noise. Also the sampling frequency and data sample block interval length can be used to affect the acquisition performance. In general, higher sampling frequency and longer data sample block interval length induce better GPS signal acquisition performance for the same received GPS signal power level.

From the simulation result, the received GPS signal at low power level, which is degraded by additive and multiplicative noise, can be detected under the condition that the received block of GPS data length is at least $1.6 \mathrm{~ms}$ and sampling frequency is at least $5 \mathrm{MHz}$.

\section{REFERENCES}

Anon. (2003). GPS Carrier-to-Noise Density. Northwood Labs LLC. http://www.northwoodlabs.com/ AN101.pdf.

Dandawate, A. V. and Giannakis, G. B. (1994) Statistical Tests for Presence of Cyclostationarity. IEEE Transactions on Signal Processing, 42(9).

Gardner, W. A. (1987). Statistical Spectral Analysis: A Nonprobabilistic Theory, Prentice-Hall, Englewood Cliffs, NJ.

Gardner, W. A. (1991). Exploitation of Spectral Redundancy in Cyclostationary Signals. IEEE Signal Processing Magazine, 8, 14-36.

Gardner, W. A. (1994). Cyclostationarity in Communications and Signal Processing. IEEE Press.

Gardner, W. A., Napolitano, A. and Paura, L. (2006). Cyclostationarity: Half a Century of Research. Signal Processing, 85: 639-697.

Ghogho, M., Ibnkahla, M. and Bershad, N. J. (1998). Analytic Behavior of the LMS Adaptive Line Enhancer for Sinusoids Corrupted by Multiplicative and Additive Noise. IEEE Transactions on Signal Processing, 46(9), 2386-2393.

Huang, P., Pi, Y. and Zhao, Z. (2009). Weak GPS Signal Acquisition Algorithm Based on Chaotic Oscillator. EURASIP Journal of Advanced Signal Processing, Vol. 2009.

Lee, J., Song, I., Kwon, H., and Kim, H.J. (2008). Locally Optimum Detection of Signals in Multiplicative and First-order Markov Additive Noises. IEEE Transactions on Information Theory, 54(1), $219-234$.

Ma, C., Jee, G. I., MacGougan, G., Lachapelle, G., Bloebaum, S., Cox, G., Garin, L. and Shewfelt, J. (2001). GPS Signal Degradation Modeling. Proceedings of the $14^{\text {th }}$ International Technical Meeting of the Satellite Division of the Institute of Navigation (ION GPS 2001), Salt Lake City, UT, 882-893.

Macabiau, C. (2000). Signal Quality Monitoring for Protection of GBAS Users Against Evil Waveforms, Proceedings of the $13^{\text {th }}$ International Technical Meeting of the Satellite Division of the Institute of Navigation (ION GPS 2000), 1202-1211.

MacGougan, G., Lachapelle, G., Nayak, R. and Wang, A. (2001). Overview of GNSS Signal Degradation Phenomena. Proceedings of the International Symposium Kinematic Systems and Geodesy, Geomatics and Navigation, 2001.

Madhani, P. H., Axelrad, P., Krumvieda, K. and Thomas, J. (2003). Application of Successive Interference Cancellation to the GPS Pseudolite Near-Far Problem. IEEE Transactions on Aerospace and Electronic Systems, 39(2), 481-488.

Middleton, D. (1999). Non-Gaussian Noise Models in Signal Processing For Telecommunications: New Methods and Results for Class A and Class B Noise Models. IEEE Transactions on Information Theory, 45(4), 1129-1149.

Progri, I. (2003). An Assessment of Indoor Geolocation Systems. Ph. D. Dissertation, Worcester Polytechnic Institute. 
Progri, I. (2011). Geolocation of RF Signals-Principles and Simulations. Springer Science \& Business Media, LLC. http://www.springer.com/engineering/electronics/book/978-1-4419-7951-3.

Progri, I. (2013). Indoor Geolocation Systems: Theory and Applications. Springer Science and Business Media, LLC (not yet available in print).

Progri, I., Bromberg, M.C. and Michalson, M. R. (2006). Maximum Likelihood GPS Parameter Estimation. Navigation, 52(4), 229-238.

Psiaki, M. L. (2001). Block Acquisition of Weak GPS signals in a Software Receiver. Proceedings of the $14^{\text {th }}$ International Technical Meeting of the Satellite Division of the Institute of Navigation (ION GPS 2001), $2838-2850$.

Song, I. and Kassam, S. A. (1990). Locally Optimal Detection of Signals in a Generalized Observation Model: the Random Signal Case. IEEE Transactions on Information Theory, 36(3), 516-530.

Song, I. and Uhm, T. C. (1991). Multiplicative Noise Model and Composite Detection, Proceedings of the IEEE, 138(6), 531-538.

Tsui, J. B. Y., (2004). Fundamental of Global Positioning System Receivers: A Software Approach. John Wiley \& Sons, Inc.

Zaikin, A. A., López, P., Baltanós, J. P., Kurths, J. and Sanjuón, M.A.F. (2002). Vibrational Resonance in a Noise-induced Structure. Physics Review, 66(1). 